\title{
La construcción sostenible a partir del empleo de ladrillos tipo PET
}

\begin{tabular}{c} 
Ing. Juan Carlos Moya MSc. ${ }^{1}$, Sr. Esteban Cevallos ${ }^{2}$, Sr. Erick Endara ${ }^{3}$ \\
${ }^{1}$ Docente - Investigador, Coordinador Unidad de Titulación Carrera de Ingeniería Civil \\
Facultad de Ingeniería, Ciencias Físicas y Matemática \\
Universidad Central del Ecuador \\
jmoya@uce.edu.ec juancmoya4@gmail.com \\
${ }^{2}$ Estudiante - Investigador, Carrera de Ingeniería Civil \\
Facultad de Ingeniería, Ciencias Físicas y Matemática \\
Universidad Central del Ecuador \\
$\begin{array}{c}{ }^{3} \text { Estudiante - Investigador, Carrera de Ingeniería Civil } \\
\text { Facultad de Ingeniería, Ciencias Físicas y Matemática } \\
\text { Universidad Central del Ecuador } \\
\text { Información del artículo } \\
\text { Recibido: julio } 2018 \\
\text { aceptado: septiembre } 2018\end{array}$ \\
\hline
\end{tabular}

\section{Resumen}

El presente proyecto investigativo demuestra la problemática en la que se encuentra el Distrito Metropolitano de Quito, esto es debido al alto consumo por parte de la sociedad de materiales plásticos, entre ellos el polietileno de tereftalato (PET), los cuales no tiene un destino final apropiado. En primer lugar el proyecto proporciona factores favorables para una construcción sostenible, porque se intenta mitigar el problema de la contaminación y la disminución del uso de mamposterías tradicionales. El objetivo es brindar a la sociedad alternativas de construcción mediante el reciclaje de materiales de alto consumo a fin de conservar el medio ambiente, una forma de mitigación es la reutilización de las botellas plásticas de cualquier capacidad como elementos constructivos de mampostería. Esta construcción novedosa debe cumplir con parámetros que brinden seguridad a sus ocupantes, para ello la investigación determinará a través de ensayos en especímenes de mampostería las bases de diseño para la construcción con elementos reciclados, verificando los resultados con las normas vigentes en el país.

Palabras clave: mampostería con ladrillo tipo pet / tracción indirecta en botellas plásticas / resistencia a la compresión de prismas / tensión diagonal en muretes / adherencia con botellas plásticas / costo mampostería de botellas tipo pet.

\begin{abstract}
Abstrac
This research project demonstrates the problems in the Metropolitan District of Quito, due to the high consumption by the society of plastic materials including polyethylene terephthalate (PET) which does not have an appropriate final destination. In the first place, the project provides favorable factors for sustainable construction, because it tries to mitigate the problem of pollution and the reduction of the use of traditional masonry. The objective is to provide society with construction alternatives through the recycling of high consumption materials to conserve the environment, a way of mitigation is the reuse of plastic bottles of any capacity as building elements of masonry. This new construction must comply with parameters that provide security to its occupants, for this the research will determine through tests in masonry specimens the design bases for the construction with recycled elements, verifying the results with the norms in force in the Country.
\end{abstract}

Keywords: Typical pet brake mamposters / indirect traction in plastic bottles / resistance to prism compression / diagonal tension in mureters / adhesion with plastic bottles / cost pet bottle mamposters. 


\section{Introducción}

El PET (polietileno de tereftalato) es uno de los residuos más abundantes mundialmente. Son elementos no retornables y tardan en degradarse entre 100 y 1.000 años. Mediante un proceso de reciclaje se puede reutilizarlo para conformar elementos aptos para la construcción, implementándolo en la creación de paredes o mamposterías logrando de esta manera una reducción significativa en la carga permanente o carga muerta de una edificación o vivienda. Al ser un elemento más liviano que los mampuestos tradicionales (ladrillos o bloques), se obtendrán estructuras más ligeras y se reducirían los costos de construcción.

El ladrillo PET podrá ser fabricado con diferentes envases o recipientes de plástico de distintas capacidades y el material de relleno (arena, tierra, espuma flex, paja, entre otros) puede variar dependiendo de la zona en donde se va a construir la vivienda. De esta manera se puede considerar como construcción sostenible debido a que reutiliza una gran cantidad de residuos de plásticos y otros elementos de sitio, evitando la explotación de materias primas a nivel de canteras para la producción de mampuestos de ladrillo o bloque.

Existe poca conciencia por parte del ser humano al momento de reciclar materiales que emplea de forma frecuente, una vez que los productos fueron consumidos, la mayoría de los envases de botellas plásticas PET son dispuestos en rellenos sanitarios, cauces, calles o tiraderos clandestinos; pero estos podrían ser reutilizados en la construcción como nuevas alternativas ecológicas, entre ellas el uso de botellas de plástico como ladrillo tipo PET en mampostería, permitiendo tener elementos sismo resistentes a bajo costo siempre y cuando participe o colabore la comunidad en la edificación de las mismas apoyando de esta manera con una alternativa para mitigar la contaminación ambiental que tanto afecta a ciudades y pueblos ecuatorianos.

En el cantón San Lorenzo de la provincia de Esmeraldas se utilizó cerca de 21.500 botellas para edificar una vivienda de aproximadamente $275 \mathrm{~m} 2$ de construcción ${ }^{1}$.

1 Celi, M., (2013). Análisis del sistema constructivo con botellas recicladas PET, y su aplicación en el diseño de un centro de exposición

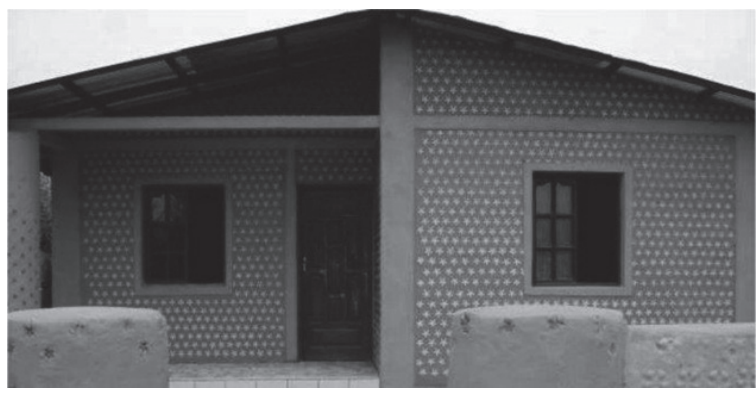

Fotografía 1. Vivienda construida con ladrillos tipo PET.

Fuente: Universidad Técnica Particular de Loja.

En el Distrito Metropolitano de Quito DMQ se generan al día 2.037 toneladas de basura o residuos sólidos urbanos RSU de los cuales el 57\% son residuos orgánicos, el $\mathbf{2 4 \%}$ es material reciclable, 19\% rechazos (papel higiénico, servilletas usadas, envases de espuma flex, envolturas de golosinas y caramelos) y un porcentaje bajo de residuos peligrosos.

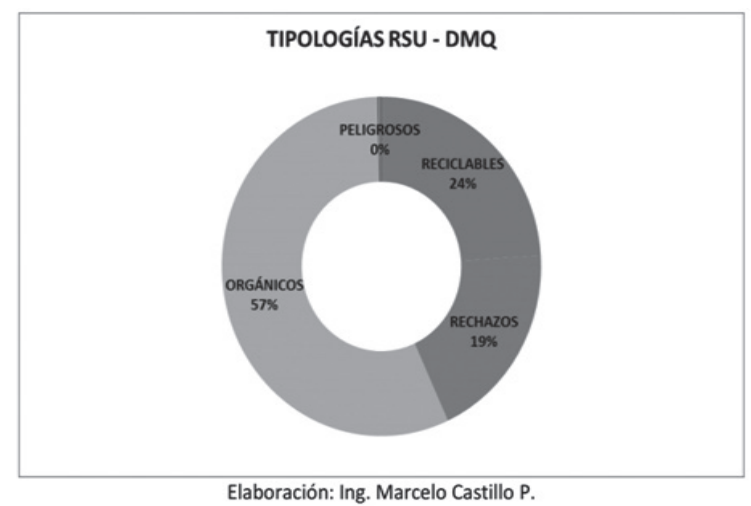

Gráfico 1. Tipologías de los residuos sólidos urbanos en el DMQ.

Fuente: Emaseo $(2016)^{2}$

De los porcentajes descritos el material reciclable contiene un $1,94 \%$ de botellas PET que represen$\tan 38,572$ ton/día se lo puede observar en el ítem quinto de la siguiente tabla.

(tesis de pregrado). Universidad Técnica Particular de Loja, Loja, Ecuador.

2 Consultoría para la realización de un estudio de caracterización de residuos sólidos urbanos domésticos y asimilables a domésticos para el Distrito Metropolitano de Quito, Recuperado de: http://www.emaseo.gob.ec/documentos/pdf/Caracterizacion residuos.pdf. 
Tabla 1. Caracterización de los residuos sólidos del cantón Quito
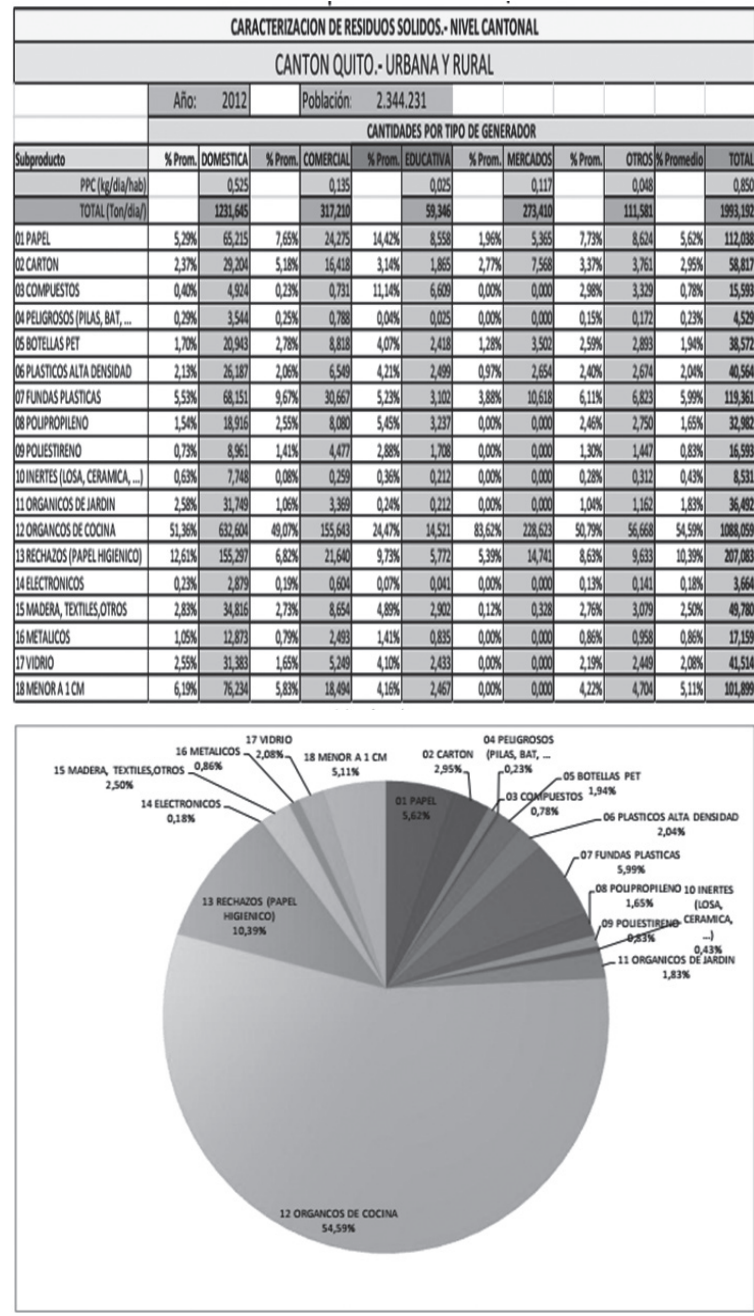

Gráfico 2. Composición de los residuos sólidos urbanos del cantón Quito

Fuente: Emaseo

Según estudios realizados por Emaseo en el DMQ con una población urbana y rural de 2'551.721 habitantes la producción o generación per cápita de residuos sólidos $\left(\mathrm{PPC}^{3}\right)$ global, se encuentra, en promedio, en un valor de $0,850 \mathrm{~kg} / \mathrm{hab} /$ día equivalentes a $319 \mathrm{~kg} / \mathrm{hab} / \mathrm{año}$; siendo $0,879 \mathrm{~kg} /$ hab/día a nivel urbano y $0,779 \mathrm{~kg} / \mathrm{hab} /$ día a nivel rural.

Se han obtenido valores sobre la generación de residuos sólidos en la capital a partir del año 2012, los cuales constan identificados en la tabla adjunta.

3 PPC: Producción per cápita de residuos sólidos: cantidad de residuos sólidos generados por persona en un día; $\mathrm{kg} / \mathrm{habitante/día.}$
Tabla 2. Caracterización de los residuos sólidos urbanos y rurales del cantón Quito

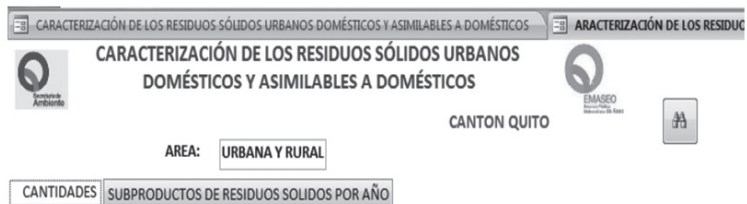

\begin{tabular}{|c|c|c|c|c|c|c|c|}
\hline \multicolumn{8}{|c|}{ CANTIDADES SUBPRODUCTOS DERESIDUOS SOLDOS POR AÑO } \\
\hline \multirow[b]{2}{*}{ AÑo } & \multirow[b]{2}{*}{ POBLACION } & \multicolumn{6}{|c|}{ CANTIDADES POR TIPO DE GENERADOR (EN TON/DIA) } \\
\hline & & DOMESTICA & COMERCIAL & EDUCATIVA & MERCADOS & OTROS & TOTAL \\
\hline \multicolumn{2}{|c|}{ PPC (kg/dia/hab) } & 0,5254 & 0,1353 & 0,0253 & 0,1166 & 0,0476 & 0,8503 \\
\hline 2012 & 2.344.231 & $1.231,645$ & 317,210 & 59,346 & 273,410 & 111,581 & $1.993,192$ \\
\hline 2013 & 2.382 .343 & $1.251,305$ & 321,443 & 60,287 & 278,039 & 113,059 & $2.024,133$ \\
\hline 2014 & 2.421 .817 & $1.271,671$ & 325,838 & 61,261 & 282,833 & 114,594 & $2.056,197$ \\
\hline 2015 & 2.462 .715 & $1.292,772$ & 330,400 & 62,271 & 287,801 & 116,186 & $2.089,431$ \\
\hline 2016 & 2.505 .063 & $1.314,625$ & 335,136 & 63,317 & 292,944 & 117,838 & $2.123,861$ \\
\hline 2017 & 2.548 .928 & $1.337,264$ & 340,050 & 64,401 & 298,271 & 119,553 & $2.159,539$ \\
\hline 2018 & 2.594 .346 & $1.360,708$ & 345,149 & 65,524 & 303,784 & 121,332 & $2.196,496$ \\
\hline 2019 & 2.641 .393 & $1.384,996$ & 350,442 & 66,686 & 309,494 & 123,180 & $2.234,798$ \\
\hline 2020 & 2.690 .028 & $1.410,105$ & 355,921 & 67,889 & 315,396 & 125,091 & $2.274,402$ \\
\hline 2021 & 2.740 .364 & $1.436,095$ & 361,602 & 69,133 & 321,505 & 127,073 & $2.315,409$ \\
\hline
\end{tabular}

Fuente: Emaseo

\section{Justificación}

\subsection{Justificación teórica}

Buscar nuevas alternativas constructivas y ecológicas que sirvan como elementos sismo resistentes a bajo costo, aprovechando materiales de la zona donde será la construcción y controlar la contaminación generada por el uso de grandes cantidades de energía para la elaboración de mampostería tradicional.

\section{Justificación práctica}

Encontrar soluciones económicas, eficientes y seguras para edificaciones de diversos usos, a través de un estudio técnico que permita conocer las propiedades físico mecánicas de un elemento de mampostería; también contar con información acerca de esta alternativa de construcción ecológica siendo un aporte nuevo para la la comunidad.

\section{Antecedentes}

En la carrera de Ingeniería Civil de la Universidad Central del Ecuador se han realizado investigaciones acerca de la aplicación del ladrillo PET como elemento para la construcción sostenible combinando botellas de distintas capacidades, diferentes elementos de relleno y varias combinaciones del mortero de pega con los mampuestos para observar la adherencia de los elementos; de 
dichos estudios se citan la condiciones más relevantes:

En una primera investigación realizada en enero de 2015 se verificó las propiedades de las mamposterías empleadas en la construcción de varias aulas en el colegio Liceo Internacional de Quito. Esta técnica la auto calificaron de manera inapropiada como antisísmica, se utilizaron botellas plásticas de 1.35 litros rellenas de arena, con la finalidad de estudiar la adherencia entre las botellas y el mortero se trabajó con botellas lisas, botellas con rugosidad y botellas con clavos diametralmente opuestos.

Al final de este se obtuvieron diferentes limitaciones tanto en la adherencia del conjunto (pasta de mortero - botellas) y un peso propio elevado $\left(570 \mathrm{~kg} / \mathrm{m}^{2}\right)$. Como recomendaciones de dicha investigación se sugiere realizar un nuevo estudio empleando botellas de menor capacidad y otro material de relleno para disminuir el peso del ladrillo PET.

En la segunda etapa de investigación realizada en mayo de 2017, se cambió el tipo de envases, empleando botellas de 0.50 litros, rellenándolas de suelo del sitio de construcción (tierra), con la finalidad de aligerar el peso propio del ladrillo PET; además se empleó un aditivo plastificante para conseguir una mejor adherencia entre el mortero y los mampuestos.

Se obtuvieron resultados favorables al disminuir el peso propio aproximadamente en un 30\% (394 $\mathrm{kg} / \mathrm{m}^{2}$ ) sin embargo, dicho valor aún sigue siendo elevado. El empleo del aditivo originó una pérdida en la resistencia del mortero, a pesar de este inconveniente se logró mejorar las propiedades físico-mecánicas del conjunto.

A partir de las ideas anteriormente expuestas, el propósito del presente estudio es conseguir un elemento que tenga un peso propio similar a los valores recomendados por la NEC 2015 (peso propio $\leq 200 \mathrm{~kg} / \mathrm{cm}^{2}$ ) y con mejores propiedades físico-mecánicas a un bajo costo; con la finalidad de alcanzar estas características en los ladrillos PET se debe saber responder a las siguientes preguntas:
¿Cuál será el material de relleno para disminuir el peso propio?

¿De qué forma se deben rellenar las botellas?

¿Cómo mejorar la adherencia entre el mortero y el ladrillo PET?

¿Qué propiedades físico-mecánicas se logran mejorar?

¿Cuál es el costo de construcción de este tipo de mampostería?

\section{Objetivos}

\subsection{Objetivo general}

- Mejorar las bases de diseño para la construcción sostenible con mampostería de ladrillo tipo PET.

\subsection{Objetivos específicos}

- Establecer las propiedades físicas y mecánicas de los elementos que conforman la mampostería optimizada.

- Comprobar la adherencia entre el mortero y el ladrillo tipo PET optimizado.

- Determinar los costos de construcción por cada metro cuadrado de mampostería tipo PET.

- Comparar los resultados con los obtenidos en las investigaciones anteriores sobre la construcción sostenible con ladrillos PET.

\section{Análisis de los materiales}

En esta tercera etapa de investigación se emplearon botellas de $600 \mathrm{ml}$ de capacidad, con una longitud de $24 \mathrm{~cm}$, diámetro promedio de la botella $6,5 \mathrm{~cm}$ y un diámetro de $2,1 \mathrm{~cm}$ en la boca del envase; las mismas fueron rellenadas de espuma flex reciclada y triturada previamente. 


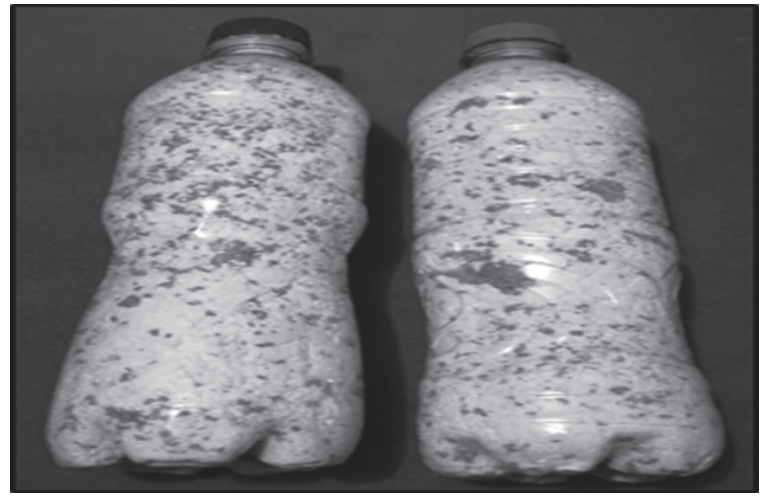

Fotografía 2. Caracterización de los ladrillos PET.

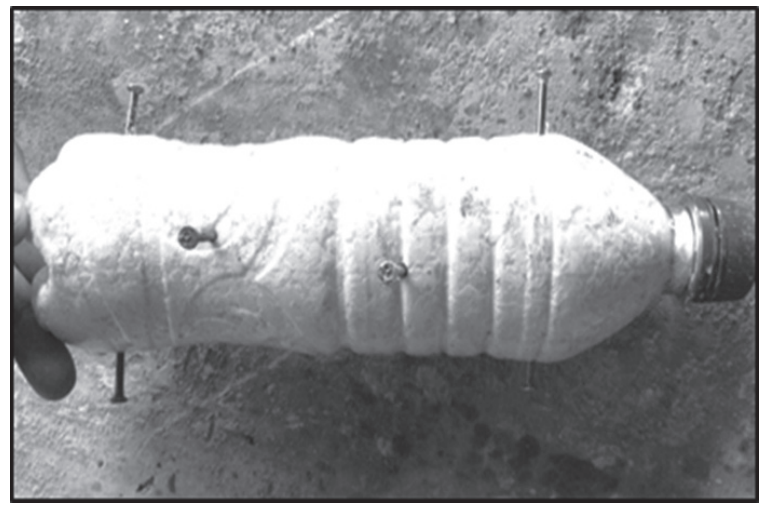

Autores: Esteban Cevallos / Erick Endara

Nuevamente en esta etapa de investigación se empleó el aditivo plastificante y como estudio complementario se utilizaron botellas con clavos diametralmente opuestos para verificar las condiciones de adherencia entre el mortero y los ladrillos PET

\subsection{Propiedades físicas de la mampostería}

Un sistema de mampostería deberá tener la capacidad para resistir las diferentes cargas a las que estará expuesta durante su vida útil como son: cargas gravitacionales, cargas sísmicas, presiones de tierra, acción de viento, entre otras; debe ser una barrera contra el ruido, contra el frío o el calor, además de resistir los daños que ocasionaría el fuego en caso de un incendio.

\subsubsection{Durabilidad}

La durabilidad es la capacidad de resistir influencias ambientales y físicas, en el transcurso del tiempo, de un material de construcción trabajando por separado o conjuntamente.
En cuanto a la mampostería, específicamente se trata de las resistencias a los cambios de las condiciones naturales como son la humedad y la temperatura a la que están expuestos. Esta capacidad de la mampostería se evalúa realizando pruebas de congelación y descongelación, consistiendo en condiciones repetitivas de saturación con ciclos de humedecimiento y secado.

Dado que la vida útil o el tiempo de descomposición de las botellas plásticas, utilizadas como ladrillo PET de esta investigación, va desde 100 a 1.000 años, se considera que este material cumple satisfactoriamente con esta propiedad.

\subsubsection{Absorción}

La absorción de una mampostería es la medida de porosidad de un bloque o ladrillo, por donde se puede filtrar algún tipo de líquido, tendiendo a la disgregación.

Debido a que el ladrillo PET está conformado por botellas plásticas, no absorberá ningún tipo de líquido de su alrededor, teniendo la característica de impermeable, evitando la disgregación del material, siendo este un gran problema en tipos de mampostería tradicional.

\subsection{Propiedades mecánicas de la mampostería}

La mampostería al estar formada por dos materiales que tienen características distintas (esfuerzo-deformación) y encontrarse sometidos a la acción de esfuerzos de compresión tienden a deformarse de diferente manera, es decir que tanto el mampuesto como el mortero de pega reaccionan indistintamente, lo que hace difícil su interacción.

Un prisma de mampostería sometido al efecto de una carga vertical, tanto el mortero como el mampuesto sufren deformaciones verticales y alargamientos transversales. Se debe destacar que, si los materiales tuviesen la oportunidad de trabajar independientemente, sus deformaciones serían distintas debido a sus respectivas propiedades elásticas.

La adherencia y las fuerzas de fricción entre las caras de contacto del mampuesto con el mortero, impiden el desplazamiento o deslizamiento rela- 
tivo lo que genera que ambos tengan una misma deformación transversal que será un intermedio de las que se inducirían por separado.

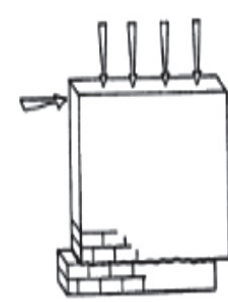

Deslizamiento

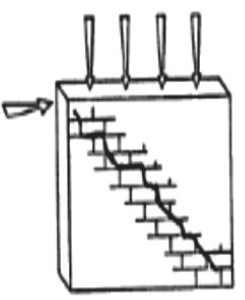

Tensión diagonal

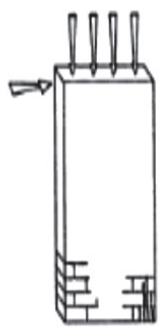

Flexocompresión

Autores: Moreno D. / Rojas S. / Gutiérrez C.

Gráfico 3. Posibles modos de falla en un muro de mampostería

El mecanismo de falla es el efecto generado en la mampostería debido a solicitaciones de compresión, corte, flexión, entre otros, por el cual se ocasionan procesos, secuencias de daño que provocan las fallas en el muro de mampostería. Cuando los muros de mampostería no cuentan con un adecuado confinamiento, una cantidad suficiente o detalle adecuado del refuerzo en los elementos confinantes, o no presentan ningún tipo de refuerzo, se han detectado diferentes tipos de patrones de agrietamiento, que dan origen a fallas en los muros.

\subsubsection{Falla por compresión axial}

Debido a la compresión axial, la falla podrá presentarse por aplastamiento de las piezas, pero este efecto también puede darse por agrietamiento vertical producido por deformaciones transversales que acompañan a la deformación longitudinal y que debido al efecto de junta pueden ser incrementadas en las piezas.

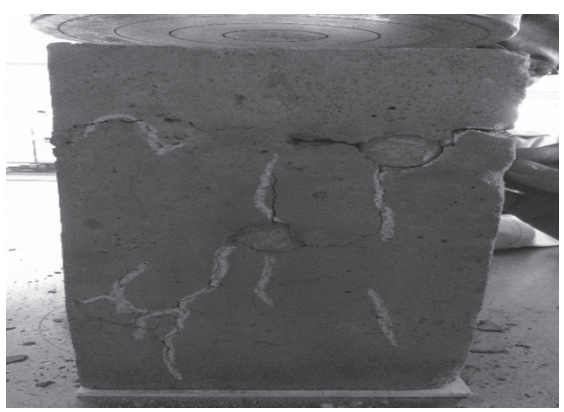

Fotografía 3. Ensayo a compresión axial de prismas de mampostería PET.

Autores: Fernando Gamboa / Andrea Recalde
Cuando el agrietamiento vertical es en exceso, este produce inestabilidad del elemento de mampostería y su falla. El aplastamiento del mortero solamente, no ocasiona, generalmente, la falla del elemento, ya que por estar colocado en capas delgadas es retenido por las piezas y no generan la inestabilidad del conjunto. (Meli R. \& Reyes A., 1992).

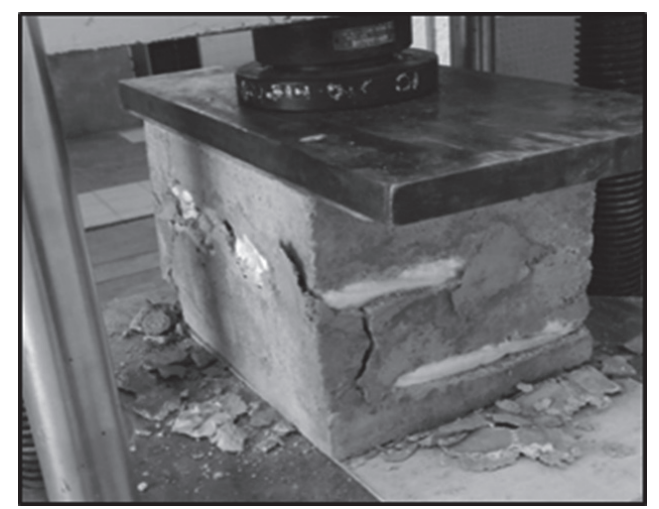

Fotografía 4. Ensayo a compresión axial de prismas de mampostería PET

Autores: Esteban Cevallos / Erick Endara

\subsubsection{Falla por tensión diagonal}

En un muro las cargas laterales inducen esfuerzos de tensión diagonal que pueden causar la falla del muro, se produce una grieta diagonal que atraviesa indistintamente de forma parcialmente recta las piezas de mampostería y el mortero, formándose desde el centro del muro, creciendo hasta los extremos del mismo.

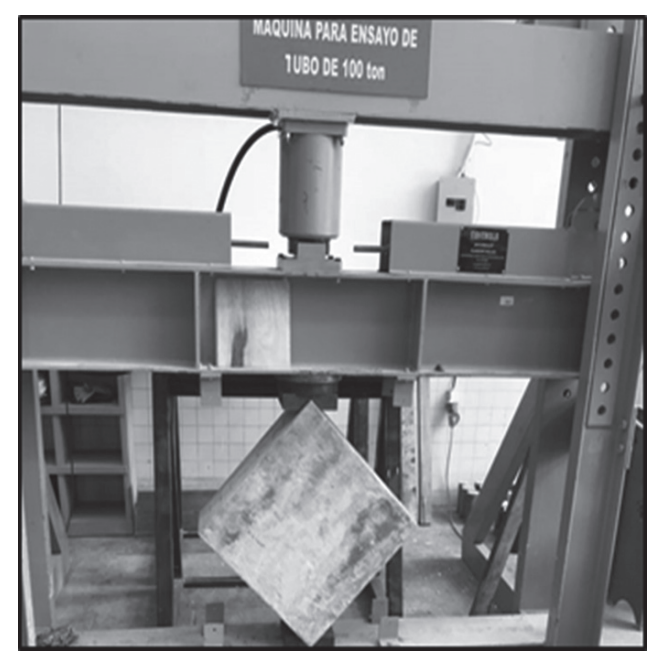

Fotografía 5. Ensayo a compresión axial de prismas de mampostería PET

Autores: Esteban Cevallos / Erick Endara 
La falla por tensión diagonal se da cuando los mampuestos son de baja resistencia y tienen buena adherencia con el mortero, de lo contrario la falla se presentaría por esfuerzos tangenciales en las juntas.

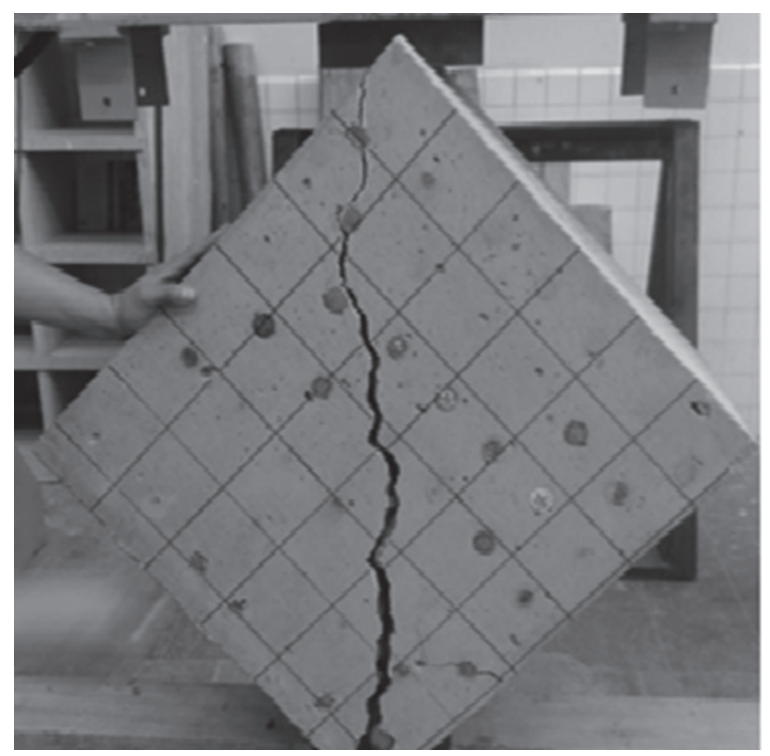

Fotografía 6. Ensayo a tensión diagonal de muretes de mampostería PET.

Autores: Fernando Gamboa / Andrea Recalde

\section{Análisis e interpretación de los resultados}

Tabla 3. Análisis comparativo de las propiedades de la mampostería PET

\begin{tabular}{|c|c|c|c|c|}
\hline PROPIEDAD & $1^{\circ}$ ETAPA & $\begin{array}{c}2^{\circ} \\
\text { ETAPA }\end{array}$ & $\begin{array}{c}3^{\circ} \\
\text { ETAPA }\end{array}$ & $\begin{array}{c}\text { ESTUDIO COM- } \\
\text { PLEMENTARIO }\end{array}$ \\
\hline $\begin{array}{l}\text { Densidad (gr/ } \\
\left.\mathrm{cm}^{3}\right)\end{array}$ & 1.75 & 1.88 & 1.26 & 1.35 \\
\hline $\begin{array}{l}\text { Peso unitaro } \\
\left(\mathrm{kg} / \mathrm{m}^{2}\right)\end{array}$ & 570.50 & 394.13 & 301.73 & 324.00 \\
\hline $\begin{array}{l}\text { Resistencia a } \\
\text { tensión dia- } \\
\text { gonal (MPa) }\end{array}$ & 0.09 & 2.22 & 0.17 & 0.25 \\
\hline $\begin{array}{l}\text { Resistencia a } \\
\text { compresión } \\
\text { axial (MPa) }\end{array}$ & 0.63 & 3.47 & 0.69 & 1.26 \\
\hline $\begin{array}{l}\text { Costo unita- } \\
\text { rio (USD /m }{ }^{2} \text { ) }\end{array}$ & 52.60 & 42.02 & 81.01 & 60.62 \\
\hline
\end{tabular}

Autores: Esteban Cevallos / Erick Endara
Luego de realizar los ensayos de laboratorio (densidad, tracción indirecta, compresión axial y tensión diagonal) en los elementos y muretes representativos de la mampostería PET, a continuación se detalla un análisis global de las diferentes etapas de la invetigación realizada en la carrera de Ingeniería Civil de la Universidad Central del Ecuador.

$$
\text { Densidad (gr/ } \left./ \mathrm{cm}^{3}\right)
$$

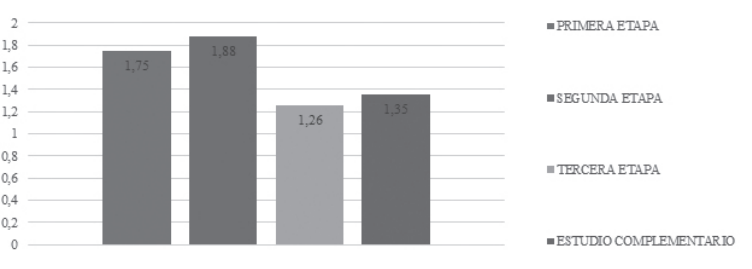

Gráfico 3. Densidad de la mampostería PET. Autores: Esteban Cevallos / Erick Endara

\subsection{Peso propio}

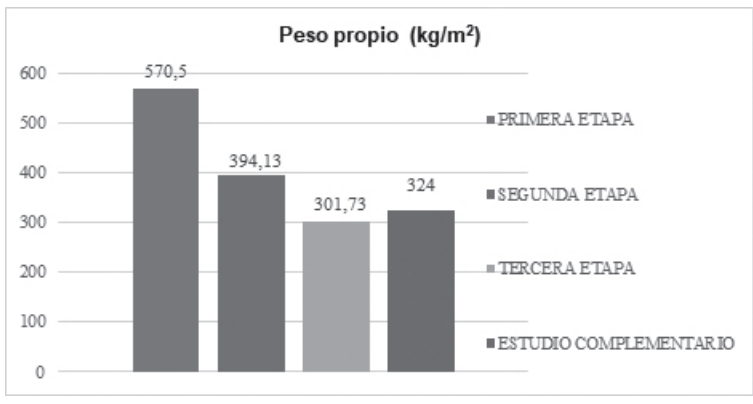

Gráfico 4. Peso propio de la mampostería PET.

Autores: Esteban Cevallos / Erick Endara

\subsection{Resistencia a la tensión diagonal}

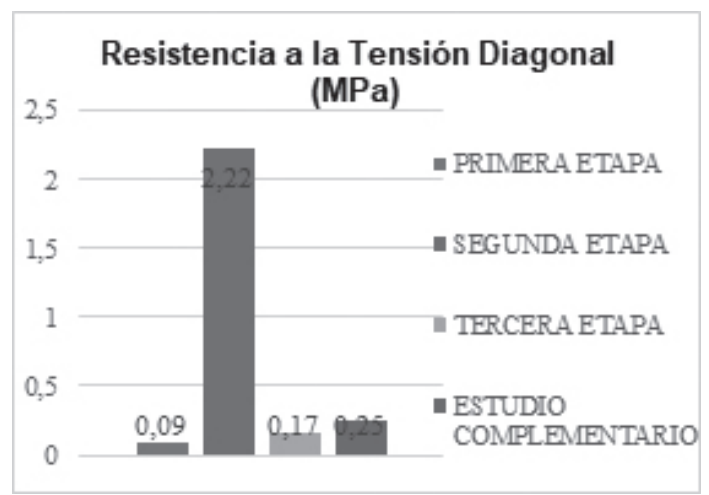

Gráfico 5. Resistencia a tensión diagonal de la mampostería PET.

Autores: Esteban Cevallos / Erick Endara 


\subsection{Resistencia a la compresión axial}

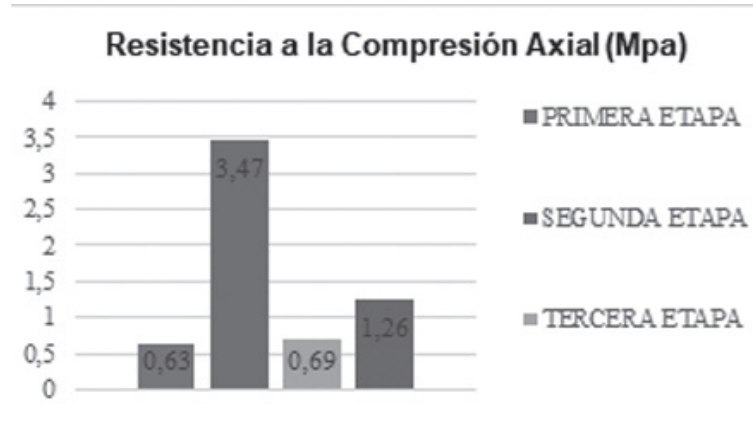

Gráfico 6. Resistencia a compresión axial de la mampostería PET.

Autores: Esteban Cevallos / Erick Endara

\subsection{Costo unitario}

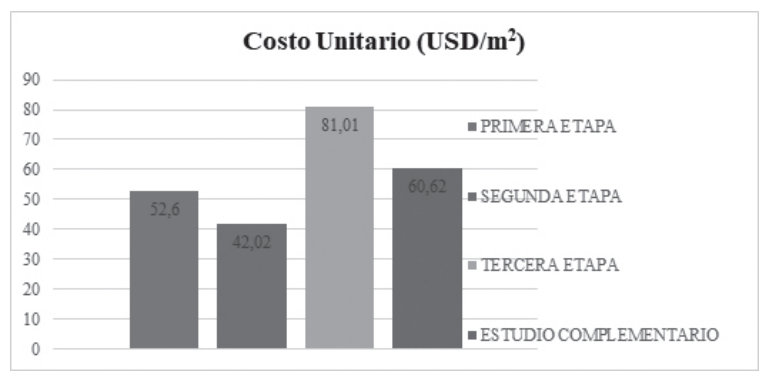

Gráfico 7. Costo Unitario de la mampostería PET.

Autores: Esteban Cevallos / Erick Endara

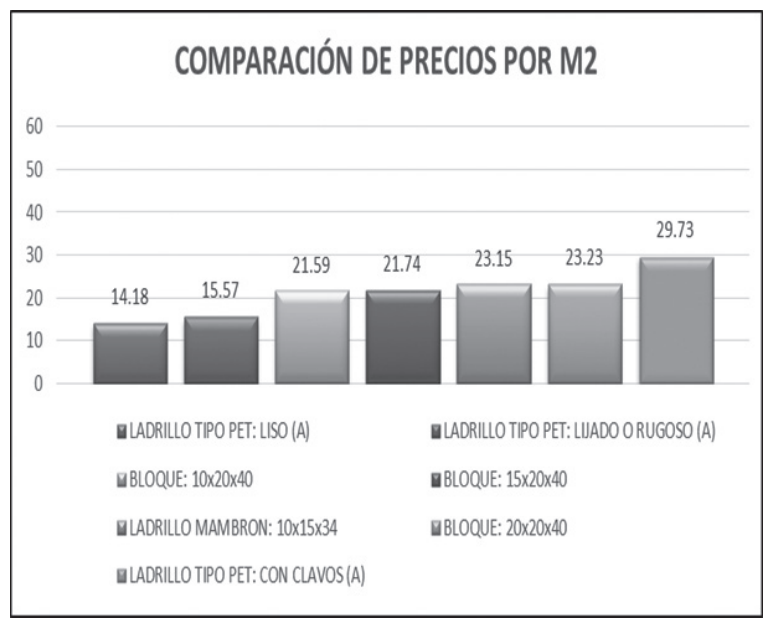

Gráfico 8. Costo unitario de diversos tipos de mampostería.

Autores: Fernando Gamboa / Andrea Recalde

\section{Conclusiones}

Los muretes construidos con botellas PET y clavos poseen menor densidad que los de la primera etapa en $22.86 \%$ y que los de la segunda etapa en $28.19 \%$, sin embargo, tiene un ligero aumento de $0.09 \mathrm{gr} / \mathrm{cm}^{3}$ que representa $7.38 \%$ de incremento al valor de los muretes construidos con elementos rellenos únicamente de espuma flex.

De igual manera el peso propio de los muretes construidos con botellas PET y clavos son más livianos en $43.21 \%$ a los de la primera etapa y son más livianos en $17.79 \%$ que los elementos de la segunda etapa y al igual que la densidad tienen un incremento del $7.38 \%$ al valor de los muretes de la tercera etapa.

Los resultados nos indican que los muretes de la segunda etapa (botellas rellenas con suelo de sitio) son los que presentan la mayor resistencia tanto en tensión diagonal 2,22 MPa como en compresión axial 3,47 MPa, en comparación a todos los demás elementos estudiados; por lo tanto se encuentra dentro de los parámetros permisibles según la NEC_SE_VIVIENDA, viviendas hasta dos pisos con luces hasta 5 metros, convirtiendo a este mampuesto en una alternativa para la construcción sostenible de viviendas.

Se evidenció que la adición de clavos diametralmente opuestos además de permitir una mejor adherencia entre el mortero y los ladrillos PET aporta en un incremento a la resistencia del elemento tanto en tensión diagonal como a la compresión axial.

Los muretes construidos en la segunda etapa son los más económicos que se obtuvieron a lo largo de esta investigación, sin embargo el costo de los muretes de la tercera etapa podría disminuir si se involucra a la comunidad en el reciclaje, acopio, limpieza y trituración del poliestireno expandido (espuma flex) que se utiliza como relleno de las botellas, de esta manera se tendría una reducción del 34,3\% del costo con una mampostería de bloque y hasta un $38,7 \%$ del costo de una mampostería de ladrillo mambrón.

El uso del aditivo plastificante con la finalidad de mejorar la adherencia entre el mortero y las bote- 
llas PET logra que la mezcla posea mayor trabajabilidad, sin embargo, ocasiona una pérdida del $25 \%$ del valor de resistencia a la compresión y no alcanza su propósito de mejorar la adherencia debido a la superficie lisa de las botellas PET.

El empleo del ladrillo PET elaborado en esta investigación, es una alternativa viable y sostenible para reducir los impactos negativos y de esta manera mitigar la contaminación ambiental, debido a que se genera un proceso de reciclaje y reutilización de dos elementos altamente contaminantes, como son las botellas PET y la espuma flex.

Finalmente, luego de analizar todos los resultados obtenidos en la presente investigación se puede afirmar que se ha mejorado la densidad, el peso propio y las resistencias a compresión axial y tensión diagonal al emplear como relleno el suelo de sitio (segunda etapa), sin embargo, la adherencia entre el mortero y las botellas PET sigue siendo una gran debilidad del sistema de mampostería propuesto.

\section{Referencias bibliográficas}

- Cabrera O. \& Ruales R. (30 de 08 de 2017). Repositorio Universidad Central. Obtenido de http://www.dspace.uce.edu.ec/bitstream/25000/12022/1/T-UCE-0011-303.pdf

- Emaseo. (2016). Empresa pública metropolitana de aseo de Quito. Obtenido de Emaseo: http://www.emaseo.gob.ec/

- Gallo Ortiz, G.O., Espino Márquez, L. I., \& Olvera Montes, A.E. (2005). Diseño Estructural de casas habitación. En Diseño estructural de casas habitación. México D.F.: McGraw-Hill.

- Gamboa F. \& Recalde A. (2015). Bases de diseño para la construcción sostenible con mampostería de ladrillo tipo PET.

- NEC. (2015). Mampostería estructural, Capítulo 10. Norma Ecuatoriana de la Construcción. 